\title{
Is inhibitory control a 'no-go' in adolescents with autism spectrum disorder?
}

\author{
Anji S Vara ${ }^{1,3,4}$, Elizabeth W Pang ${ }^{2,4}$, Krissy AR Doyle-Thomas ${ }^{3}$, Julie Vidal ${ }^{5}$, Margot J Taylor ${ }^{1,2,4}$ \\ and Evdokia Anagnostou $2,3,4,6^{*}$
}

\begin{abstract}
Background: Autism spectrum disorder (ASD) refers to a range of neurodevelopmental conditions characterized by social communication deficits, repetitive behaviours, and restrictive interests. Impaired inhibition has been suggested to exacerbate the core symptoms of ASD. This is particularly critical during adolescence when social skills are maturing to adult levels. Using magnetoencephalography (MEG), we identified the location and timing pattern of neural activity associated with inhibition in adolescents with autism, compared to typically developing adolescents.
\end{abstract}

Methods: The MEG data from 15 adolescents with ASD and 15 age-matched controls (13 to 17 years) were collected during a go/no-go task with inverse ratios of go/no-go trials in two conditions: an inhibition condition (1:2) and a baseline condition (2:1). No-go trials from the two conditions were analyzed using beamformer source localizations from 200 ms to 400 ms post-stimulus onset. Significant activations were determined using permutation testing.

Results: Adolescents with ASD recruited first the right middle frontal gyrus (200 to $250 \mathrm{~ms}$ ) followed by the left postcentral gyrus (250 to $300 \mathrm{~ms}$ ) and finally the left middle frontal and right medial frontal gyri (300 to $400 \mathrm{~ms}$ ). Typically developing adolescents recruited first the left middle frontal gyrus (200 to $250 \mathrm{~ms}$ ), followed by the left superior and inferior frontal gyri ( 250 to $300 \mathrm{~ms}$ ), then the right middle temporal gyrus (300 to $350 \mathrm{~ms}$ ), and finally the superior and precentral gyri and right inferior lobule (300 to $400 \mathrm{~ms}$ ).

Conclusions: Adolescents with ASD showed recruitment limited largely to the frontal cortex unlike typically developing adolescents who recruited parietal and temporal regions as well. These findings support the presence of an atypical, restricted inhibitory network in adolescents with ASD compared to controls.

Keywords: Autism spectrum disorder, Adolescence, Brain imaging, Inhibition

\section{Background}

It is theorised that impaired inhibitory control exacerbates the social deficits or repetitive behaviours and restricted interests [1-3] characterizing autism spectrum disorders (ASD) [4]. Inhibition is the ability to suppress a prepotent response and is one of many executive functions that aid in behavioural control. Relying on the prefrontal cortices of the brain, inhibition works in concert with other execution functions, such as working memory and attention, to exert top-down control on behaviour (in contrast,

\footnotetext{
* Correspondence: eanagnostou@hollandbloorview.ca

${ }^{2}$ Neurology, The Hospital for Sick Children, University of Toronto, 555

University Avenue, M5G 1X8 Toronto, Ontario, Canada

${ }^{3}$ Holland Bloorview Kids Rehabilitation Hospital, University of Toronto, 150

Kilgour road, M4G1R8 Toronto, Ontario, Canada

Full list of author information is available at the end of the article
}

stimulus-driven behaviours are referred to as bottom-up processing) $[5,6]$. Inadequate top-down control in individuals with ASD could manifest as behaviour that is contextually inappropriate yet self-gratifying (for example, fixating on topics and objects of specific interests or repeating motor mannerisms that provide stimulation) and be indicative of impaired inhibition.

The extent of inhibitory impairment in ASD is not well understood, as the existing literature has yielded inconsistent findings. Some behavioural studies have reported inhibition deficits in ASD [1,7-11], while others have found no impairment [12-17]. This inconsistency may, in part, be due to the variety of tasks employed and the differences in task-demands [18]. These include the Stop-signal task [19], the Stroop task $[15,20,21]$ and the anti-saccade task [9]. 
Neuroimaging studies have attempted to clarify the variability seen in behavioural studies by identifying possible endophenotypes within the brain. In typically developing individuals, particularly adults, the anterior cingulate cortex (ACC) and the right inferior frontal cortex have been implicated in inhibition tasks, likely related to their respective roles in error processing and top-down control. For example, Tamm et al. found more variability of overall brain activity in children aged 8 to 12 years compared to adults [22]. Typically developing adolescents however have been shown to activate a similar network to adults, but at a lower threshold [23].

Imaging studies on inhibition in ASD are limited, and are mainly completed in adults [24-28]. The findings from these investigations have suggested atypical overall activation patterns; however, the question remains about potential developmental differences between individuals with and without ASD. To date, only a few studies have been conducted with children [29,30] and adolescents [31]. Using a 'Preparing to Overcome Prepotency' task in functional Magnetic Resonance Imaging (fMRI), it was found that adolescents with ASD activated the left inferior prefrontal cortex, as well as parietal and occipital cortex significantly less than typically developing adolescents [31]. In contrast, ASD adults were reported to activate the left inferior frontal gyrus to a greater degree than control adults [24] during an fMRI go/no-go task. Also using a go/no-go task, Lee et al. examined the functional connectivity with fMRI of the right and left inferior frontal gyri with regions in the frontal, striatal and parietal cortices in children aged 8 to 12 years as well as adults [29]. A trend towards an interaction between age and connectivity was found between the right inferior frontal gyrus and other regions (right caudate and bilateral supplementary premotor area) in ASD [29]. In these regions, connectivity decreased with age in ASD, while in controls no change was observed with age. More recently, a magnetoencephalography (MEG) study employing a go/no-go task found decreased theta power in the anterior cingulate cortex (ACC) in children aged 7 to 14 years with ASD compared to control children [30]. Decreased ACC activity has been associated with decreased attention and poorer performance on cognitive tasks [32,33], consistent with the poorer performance seen in the ASD group of children [30].

Thus, studies have implicated the ACC and inferior frontal cortex in inhibitory control both in individuals with and without ASD. However, regions such as the parietal lobes, striatum and premotor cortex have been reported as well, but there is less of a consensus on the relevance of these regions to inhibition versus processing associated with other demands of the experimental task. As such, studies are needed to carefully characterize the inhibitory network, as well as to examine differences between adolescents with and without ASD.
Given the importance of timing in inhibition studies (for example, controlling impulsivity and anticipatory responses), neuroimaging modalities that allow measurement of the timing of brain activity add an invaluable dimension; MEG allows the determination of both the spatial and temporal patterns of brain activity [34]. Using MEG to resolve the spatiotemporal brain dynamics of inhibitory control, we adapted a go/no-go paradigm from Vidal et al. [35]. This paradigm had simple rules so as not to implicate working memory, and a very fast presentation rate to prevent ceiling effects, and to keep adolescents engaged and challenged during task performance. Using this go/no-go task, we have demonstrated that typically developing adolescents recruited a network that was spatiotemporally different from adults to perform the task at a comparable level (Vara et al., 2013; in submission).

The aims of the current study were twofold:

1. to examine differences in inhibitory control (performance measures) between adolescents with ASD and those without. We hypothesized poorer inhibition-related task performance would be seen in the ASD group compared to the control group

2. to determine the spatial and temporal brain activity associated with inhibition in adolescents with and without ASD and to compare the neural activity pattern between groups. We hypothesized that ASD adolescents would show spatially atypical and temporally delayed neural activity patterns compared to controls.

\section{Methods}

\section{Participants}

Included in this study were 30 adolescents. Fifteen participants (12 males) had an ASD diagnosis, between the ages of 13 and 17 years (mean age $15.5 \pm 1.2$ yrs; mean IQ $103.8 \pm 13.6)$. These participants were age- and sexmatched with typically developing control adolescents ( $\mathrm{n}=15 ; 12$ males; mean age $15.6 \pm 1.3$ yrs; mean IQ $112.4 \pm 10.3$; the control sample was included in a normative, developmental paper, Vara et al., in submission). None of them were on any psychotropic medications. Exclusion criteria were history of neurological disorder, including epilepsy and or acquired brain injury, known neurodevelopmental syndromes (for example, Fragile-X, tuberous sclerosis), primary psychiatric disorders (aside from ASD, for example, schizophrenia, bipolar, panic disorder), or chronic medical disorders (for example, sickle cell disease, cardiac problems, any form of cancer), current psychotropic medication use, prematurity, uncorrected vision, full scale IQ $<80$ and standard contraindications to MEG and MRI imaging (ferromagnetic objects in the body). All participants were recruited based on known ASD diagnosis from a clinician and the diagnosis was 
confirmed using the Autism Diagnostic Observational Schedule - Generic (ADOS-G) [36], and the Autism Diagnostic Interview Revised (ADI-R) [37]. Mean and standard deviation of ADOS and ADI scores for the ASD group were as follows: $\mathrm{ADI}_{\text {social: }}$ 19.4(6.4), $\mathrm{ADI}_{\text {communication }}: 14.8$

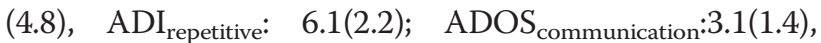

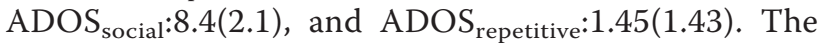
imaging study was approved by the institutional REB at Holland Bloorview Kids Rehabilitation Centre and the Hospital for Sick Children; the imaging was conducted at the Hospital for Sick Children. Informed consent or assent was obtained from all participants/guardians, as per institutional policies.

\section{Characterization measurements}

Autism Diagnostic Observation Schedule-Generic (ADOS-G)

The ADOS-G [36] is a well-validated, semi-structured clinical assessment, designed to facilitate the diagnosis of ASD (Lord et al., [36]). It has excellent psychometric properties. Research team members with established research reliability administered either module 3 or module 4 of the ADOS-G to all of the ASD participants.

Autism Diagnostic Interview - Revised (ADI-R)

This well-established semi-structured interview [37], validated for the diagnosis of ASD, was administered by a research-reliable team member. The interview, containing 93 items, generates scores in three domains: social interaction, communication and language, and repetitive or restricted interests and behaviours.

Wechsler Abbreviated Scales of Intelligence (WASI)

Two subtests of the WASI (vocabulary and matrix reasoning) were used to obtain an abbreviated measure of IQ [38]. The two-subtest WASI is a recognized version of the WASI with comparable validity in IQ estimation to the full four-subtest WASI. The two-subtest WASI was chosen to better accommodate the participants as it allows for shorter research appointments, and therefore performance endurance was more consistent across subjects.

\section{Paradigm}

The go/no-go task was adapted from a previous study [35] and validated in control adolescents and adults (Vara et al., 2013, in submission). Participants completed a 'go/no-go' task while lying in the MEG. Subjects were instructed to rapidly respond to go stimuli, solid black shapes on a white background and withhold their response to no-go stimuli, the same as Go stimuli, but with a grey ' $\mathrm{X}$ ' superimposed in the centre (Figure 1). Two conditions were run in counterbalanced order: a baseline condition with $67 \%$ no-go trials, not encouraging a tendency to respond, and an inhibition condition with $33 \%$ no-go trials, which promoted a prepotent response tendency. To equate the behavioural performance on our task (specifically, the accuracy) across our groups, we used adaptive interstimulus intervals (ISIs) that were dependent on performance. Starting at $500 \mathrm{~ms}$ ISI for the first trials, the ISI was adjusted every five stimuli, where three errors or more on no-go trials would cause the ISI to increase by $100 \mathrm{~ms}$, while fewer than three errors decreased the ISI by $100 \mathrm{~ms}$, with the minimum ISI set to $300 \mathrm{~ms}$. The stimulus duration was $200 \mathrm{~ms}$.

\section{Behavioural measures}

The mean reaction time (RT) was measured for go trials, from stimulus onset to the initial button response. RTs under $100 \mathrm{~ms}$ were not included, as they probably reflected subject anticipation. False alarm rate ( that is, percentage of commission errors) was calculated as the percentage of incorrect responses to no-go trials out of the total number of no-go trials. Hit rate was calculated as the percentage of correct go trials out of the total number of go trials. Statistical analyses on behavioural data were carried out using STATISTICA, Version 8, www.statsoft.com. A repeated measures multivariate ANOVA was run on each of the three behavioural measures - RT, false alarm rate and hit rate - to compare diagnostic group (ASD versus control) and condition type (inhibition versus baseline) effects and interactions on behavioural measures of the go/no-go task.

\section{Neuroimaging}

MEG data were acquired on a 151-channel CTF system, using a $600 \mathrm{~Hz}$ sampling rate, and an online 0 to $150 \mathrm{~Hz}$ bandpass filter, with third order spatial gradient noise cancellation. All participants also completed an MRI scan, on a 3 T Siemens Trio system. Anatomical T1-weighted MRIs (3D MPRAGE sequence: TR/TE $=2300 / 2.96 \mathrm{~ms}$; $\mathrm{FA}=9^{\circ} ; \mathrm{PAT}, \mathrm{GRAPPA}=2 ; \mathrm{FOV}=28.8 \times 19.2 \mathrm{~cm}, 1 \mathrm{~mm}$ isotropic voxels) were used to co-register the MEG data. Functional analyses were conducted with scripts written in-house that generated global field power (GFPs), source localizations and permutation tests between conditions and between groups.

\section{Magnetoencephalography analyses}

MEG trials were epoched into 600-ms windows with a 100-ms pre-stimulus baseline. Only correct no-go trials were analysed. Trials with artefacts such as eye blinks and movement were manually removed on a trial-by-trial basis, based on the agreement of two research members. The no-go trials (approximately 100 trials per participant) were averaged, and then grand averaged across participants, by condition (baseline versus inhibition) and group.

Global field power (GFP), which is the root mean squared power across all sensors, was calculated on our grand-averaged data, to determine MEG amplitude changes across time. To better visualize the frontal differences between groups, we generated GFP plots from the 38 frontal sensors (Figure 2). Based on the timing of the peak 


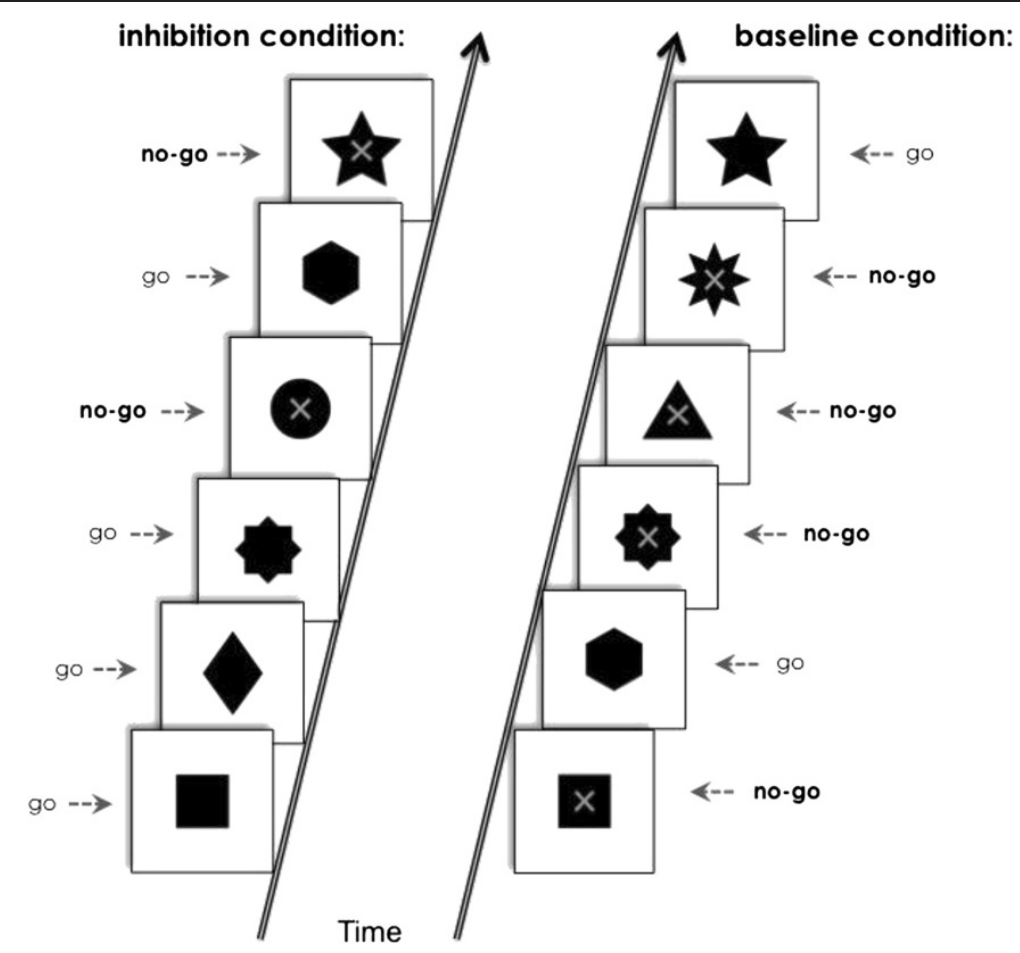

Figure 1 Illustration of the go/no-go paradigm employed in this study, with the inhibition condition on the left (consisting of $33 \%$ no-go trials) and the baseline condition on the right (consisting of $\mathbf{6 7 \%}$ no-go trials). The 'Go' stimuli, seen as solid black shapes, and the 'no-go' stimuli, seen as black shapes with an X superimposed on them, are labelled.

difference observed in these GFP plots, as well as our expectations from our normative study, in which activity related to inhibition was observed after $200 \mathrm{~ms}$, we analysed the MEG data between 200 and $400 \mathrm{~ms}$.

For source localisation analyses, we used an in-house vector beamformer algorithm (SPF) [39]. Multisphere headmodels were created from initial fiducial positions co-registered to each individual's T1 MRI [40]. Data were filtered using a bandpass of 0.5 to $30 \mathrm{~Hz}$ and beamformer images were calculated over 50-ms non-overlapping time intervals, from 200 to $400 \mathrm{~ms}$, for each condition and group. The resultant images with a spatial resolution of $5 \mathrm{~mm}$ were normalized to an MRI template with SPM2 (Wellcome Department of Imaging Neuroscience, London, UK). The 3D baseline condition (67\% no-go) images were subtracted from those from the inhibition condition $(33 \%$ no-go) to remove the visual activity [41,42].

To assess significance of within-group activity, permutation tests were completed on the subtracted beamformer images (2,048 permutations), generating activation maps at $P<0.005$. Permutation tests shuffled group membership of the two samples, while maintaining the cardinality, and computing mean differences between the samples. The calculated mean differences for $n>2,000$ permutations were plotted to create a distribution, allowing the difference from the original sample to be compared to the distribution of values, to obtain $P$ values. Between-group differences on the beamformer images were tested as well using permutation tests.

Images with $P<0.005$ were visualised using MRI3DX, and only the highest $15 \%$ of peak activations were included, and with their Talairach coordinates noted; anatomical terms and Brodmann areas (BA) were verified in Talairach Client $[43,44]$.

\section{Results}

\section{Behavioural results}

Mean RT, hit rate and false alarm rate are listed in Table 1 for both condition types and group types.

\section{Reaction time}

There were no main effects of condition (inhibition or baseline conditions) $(\mathrm{F}(1,28)=0.05, P=0.825)$, diagnostic group (control or ASD) $([\mathrm{F}(1,28)=1.937, P=0.175)$ or an interaction $(\mathrm{F}(1,28)=2.478, P=0.127)$ for RT.

\section{False alarm rate}

A main effect of condition type was found $(\mathrm{F}(1,28)=79.554$, $P=0.001)$ where the inhibition condition $(\mathrm{M}=22.3 \% \pm 12.6$ $\mathrm{SD}$ ) evoked a higher false alarm rate than the baseline condition $(\mathrm{M}=5.2 \% \pm 4.6 \mathrm{SD})$. A trend towards a main effect of diagnostic group on false alarm rate was also seen $(\mathrm{F}(1,28)=3.875, P=0.059$; eta squared $=0.122)$, where higher false alarm rates were seen in adolescents with 


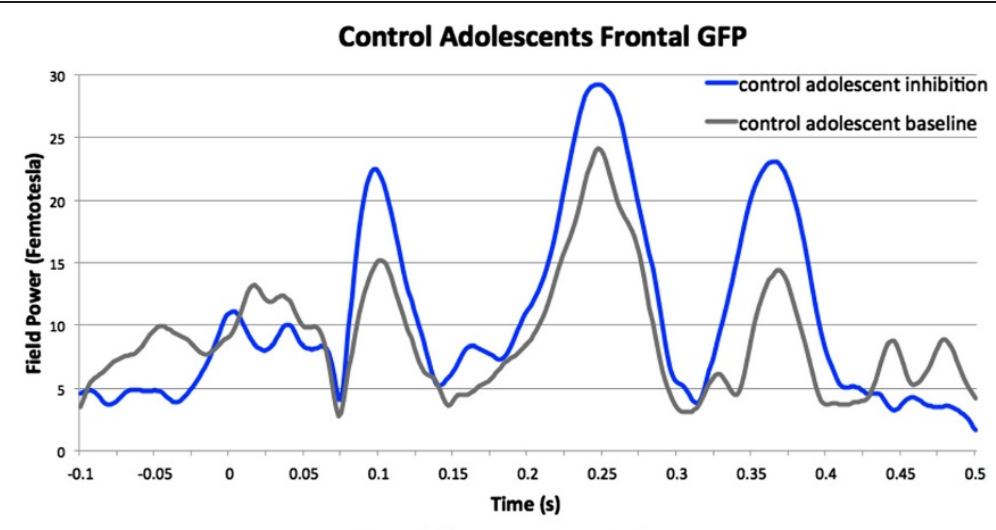

ASD Adolescents Frontal GFP
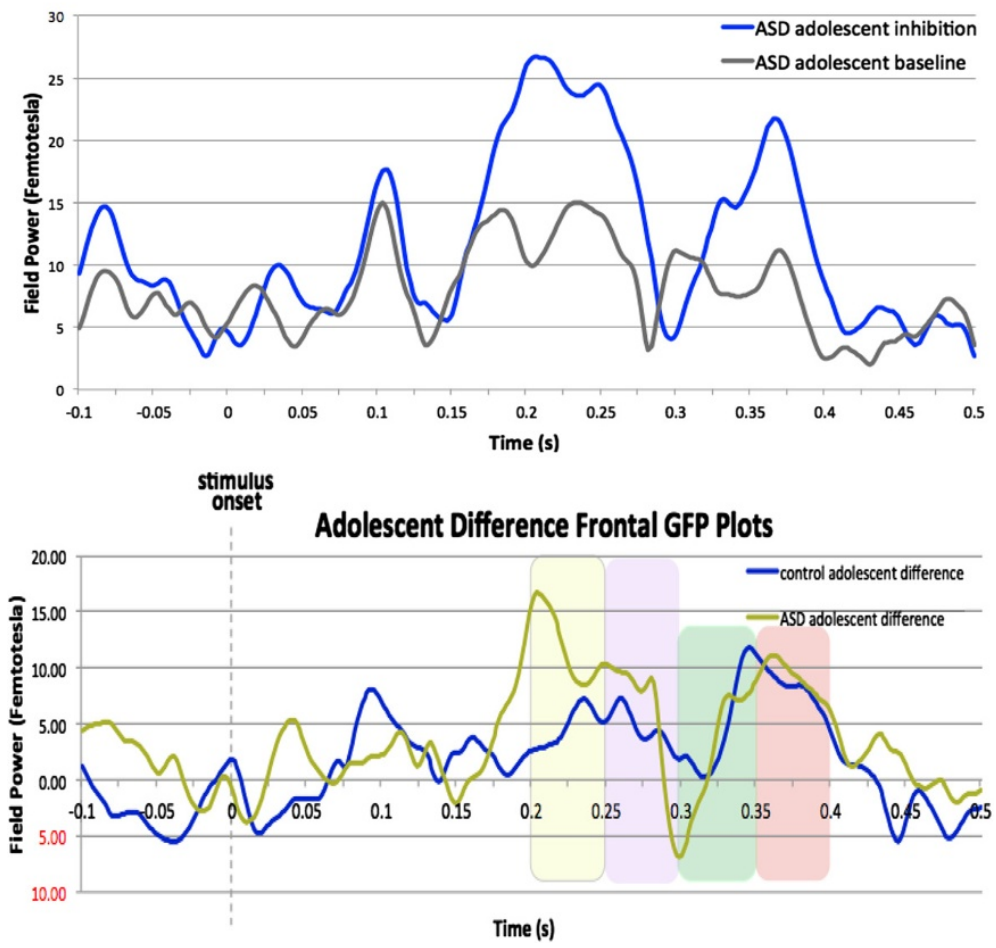

Figure 2 Global field power plots from frontal sensors. Upper plots: Global field power (GFP) plots from the frontal magnetoencephalography (MEG) sensors for the inhibition trials for the two conditions in the control adolescents (top plot) and the adolescents with autism spectrum disorder (ASD) (middle plot). Lower plot: Difference waveforms for Global field power (GFP) between the inhibition and baseline conditions, measured by MEG sensors over the frontal area of the brain on correct no-go trials for the two groups: control (blue) and ASD (gold). Stimulus onset is marked at 0 seconds. Four 50 ms time windows of interest are marked.

ASD $(M=16.4 \% \pm 8.7 \mathrm{SD})$ than controls $(\mathrm{M}=11.1 \% \pm 7.8$ $\mathrm{SD})$. The group by condition interaction was not significant $(P=0.110)$. However, because the interaction was expected to be nonsignificant for the baseline condition, we evaluated the group by condition interaction for the no-go condition. The effect of group on the false alarm rates for the no-go condition was examined and a trend towards a main effect of diagnostic group was found for the no-go condition $[((1,28)=3.737, P=0.63$; eta squared $=.0118)$, where higher false alarm rates were seen in adolescents with ASD (M = $26.5 \% \pm 3.1 \mathrm{SD})$ than in control teens $(\mathrm{M}=18.1 \% \pm 3.1 \mathrm{SD})$.

\section{Hit rate}

There were no main effects of condition type $(\mathrm{F}(1,29)=$ 0.839, $P=0.367)$, diagnostic group $(\mathrm{F}(1,28)=1.063, P=$ $0.311)$ or an interaction $(\mathrm{F}(1,29)=2.584, P=0.119)$ for hit rate.

\section{Magnetoencephalography results} Time course of global brain activity

Waveforms representing the magnitude of overall frontal brain activity occurring from $100 \mathrm{~ms}$ prior to stimulus onset to 500-ms post-stimulus onset are plotted in Figure 2. 
Table 1 Behavioural measures for go/no-go task for autism spectrum disorder (ASD) and control participants: mean and standard deviation (SD) for reaction time (RT), hit rate (Hit), and false alarm rate (FA)

\begin{tabular}{lllll}
\hline & Condition & $\overline{\mathbf{x}} \mathbf{R T}(\mathbf{s})$ & Hit (\%) & FA (\%) \\
\hline ASD & Inhibition & $361 \pm 122$ & $93.0 \pm 14.3$ & $26.5 \pm 13.2$ \\
& Baseline & $342 \pm 50$ & $88.7 \pm 18.8$ & $6.3 \pm 4.1$ \\
Controls & Inhibition & $309 \pm 34$ & $94.9 \pm 10.8$ & $18.1 \pm 10.7$ \\
& Baseline & $335 \pm 34$ & $96.1 \pm 3.6$ & $4.1 \pm 4.8$ \\
$\begin{array}{l}\text { Effect size of } \\
\text { group difference } \\
\text { (eta squared) }\end{array}$ & Inhibition & 0.042 & 0.008 & 0.167 \\
\hline
\end{tabular}

These waveforms display differences of mean global field power (GFP) measured between-conditions for frontal sensors. These plots show the difference between the inhibition and baseline conditions for the control (in blue) and ASD (in gold) groups and peak differences guided our selection of time windows of interest for further analyses.

\section{Timing and localisation of neural activity}

Significant within-group activations, where the inhibition condition was significantly greater than the baseline condition $(P<0.005$, uncorrected; $\geq 5 \mathrm{~mm}$ from peak activation) are listed in Table 2 (with one sample, one-tailed permutation tests completed on inhibition minus baseline condition event-related beamforming (ERB) images; >2000 permutations; top 15\% chosen) and displayed in Figure 3. No between-group differences were found in areas of interest (unpaired, two sample, two-tailed permutation tests, $P<0.005$, uncorrected, 5,000 permutations).

Control adolescents activated the left middle frontal gyrus (BA 6) from 200 to $250 \mathrm{~ms}$ followed by the left superior frontal gyrus (also BA 6) and left inferior frontal gyrus (BA 45) from 250 to $300 \mathrm{~ms}$. From 300 to $350 \mathrm{~ms}$, the typically developing adolescents recruited the right middle temporal gyrus (BA 21) and the right superior temporal gyrus (BA 22) from 350 to $400 \mathrm{~ms}$. The right precentral gyrus (BA 4) and right inferior parietal lobule (BA 40) were also recruited during this final time window (350 to $400 \mathrm{~ms}$ ).

The adolescents with ASD first recruited the right middle frontal gyrus (BA 45) from 200 to $250 \mathrm{~ms}$ followed by the left postcentral gyrus (BA 3) from 250 to $300 \mathrm{~ms}$. The left middle frontal gyrus (BA 10) was then activated from 300 to $400 \mathrm{~ms}$, along with the right medial frontal gyrus from 350 to $400 \mathrm{~ms}$.

\section{Discussion}

A diagnosis of ASD in our adolescent sample was associated with poorer impulsivity measures as seen in higher false alarm rates, during the inhibition condition of our task compared to the baseline condition. Neuroimaging findings complemented these behavioural findings by revealing an inhibitory network that differed significantly from the typically developing adolescent group. Adolescents with ASD recruited predominantly the frontal cortex, while controls recruited frontal as well as supplementary regions, including the inferior parietal lobule and the temporal lobe. Additionally, the ASD group initially engaged the right rather than the left frontal cortex and activated an area in the prefrontal cortex (BA 10).

\section{Behavioural measures across groups}

No significant differences $(P<0.05)$ were found between groups for response accuracy, measured by hit rate or RT. An ASD diagnosis was associated, however, with a trend

Table 2 Areas of activation $(P<0.005)$ during time windows of interest in control and autism spectrum disorder (ASD) adolescents

\begin{tabular}{|c|c|c|c|c|c|c|}
\hline \multirow{2}{*}{$\frac{\text { Time window }}{200 \text { to } 250 \mathrm{~ms}}$} & \multirow[b]{2}{*}{$L$} & \multirow{2}{*}{$\begin{array}{l}\text { Anatomical area } \\
\text { Middle frontal gyrus }\end{array}$} & \multirow{2}{*}{$\frac{B A}{6}$} & \multicolumn{3}{|c|}{ Talairach coordinate $(x, y, z) s$} \\
\hline & & & & -25 & -5 & 55 \\
\hline \multirow[t]{2}{*}{250 to $300 \mathrm{~ms}$} & $L$ & Superior frontal gyrus & 6 & -20 & 5 & 70 \\
\hline & $\mathrm{L}$ & Inferior frontal gyrus & 45 & -55 & 25 & 15 \\
\hline 300 to $350 \mathrm{~ms}$ & $\mathrm{R}$ & Middle temporal gyrus & 21 & 50 & 0 & -10 \\
\hline \multirow[t]{3}{*}{350 to $400 \mathrm{~ms}$} & $\mathrm{R}$ & Precentral gyrus & 4 & 50 & -10 & 50 \\
\hline & $\mathrm{R}$ & Superior temporal gyrus & 22 & 50 & 5 & -5 \\
\hline & $\mathrm{R}$ & Inferior parietal lobule & 40 & 40 & -50 & 45 \\
\hline Time window & & Anatomical area & BA & \multicolumn{3}{|c|}{ Talairach coordinates $(x, y, z)$} \\
\hline 200 to $250 \mathrm{~ms}$ & $\mathrm{R}$ & Middle frontal gyrus & 46 & 50 & 25 & 25 \\
\hline 250 to $300 \mathrm{~ms}$ & $L$ & Postcentral gyrus & 3 & -55 & -20 & 40 \\
\hline 300 to $350 \mathrm{~ms}$ & $L$ & Middle frontal gyrus & 10 & -30 & 45 & 5 \\
\hline \multirow[t]{2}{*}{350 to $400 \mathrm{~ms}$} & $\mathrm{R}$ & Medial frontal gyrus & & 5 & 60 & 0 \\
\hline & $L$ & Middle frontal gyrus & 10 & -30 & 50 & 5 \\
\hline
\end{tabular}




\section{Peak activations:CTRL Adolescents ASD Adolescents}

a)

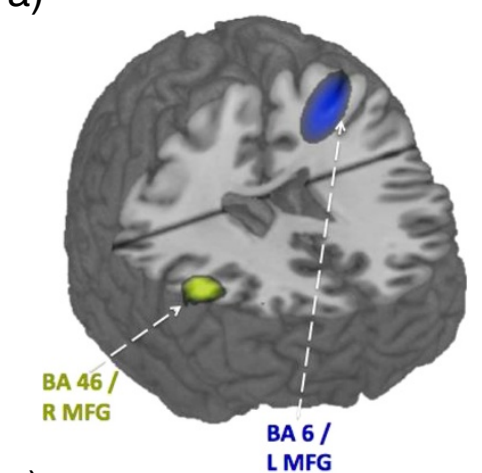

c)

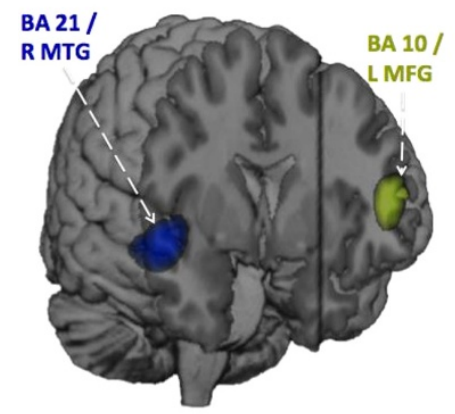

b)

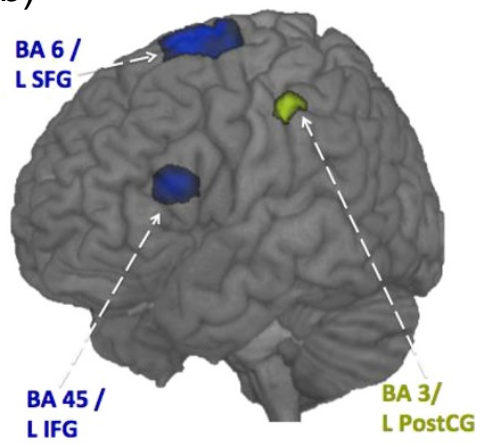

d)

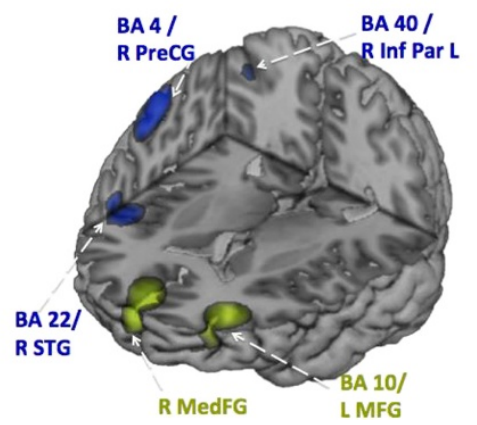

Figure 3 Locations of significant $(P<0.005)$ neural activations for both autism spectrum disorder (ASD) (green) and control adolescents (blue), where the inhibition condition was greater than the baseline condition, across time windows of interest. a) 200 to 250 ms b) 250 to 300 ms c) 300 to 350 ms d) 350 to 400 ms. L, left; R, right; G, gyrus; IMG, middle frontal; SFG, superior frontal; IFG, inferior frontal gyrus; MedFG, medial frontal gyrus; PostC G, postcentral gyrus; PreC G, precentral gyrus; Inf Par L, inferior parietal lobule; MTG, middle temporal gyrus; STG, superior temporal gyrus.

for higher false alarm rates $(P=0.059)$, or commission errors, suggesting poorer inhibitory skills in ASD individuals. A significant main effect between the baseline and inhibition conditions for false alarm rates $(P=0.001)$ confirms that the baseline condition was an effective control task, as it incurred a lower error rate.

\section{Magnetoencephalography results across groups Source localization: comparing adolescents with and without ASD}

The detection of activations in this MEG study was datadriven and done on the whole-brain, thus reflecting activity in regions without a bias of prescribed ROIs. Differences between our results and those in the literature may be due in part to our analysis approach, which contrasted no-go trials in our inhibition and baseline conditions, whereas other studies contrasted no-go trials against go trials. Our method allowed for the investigation of inhibitory control without the confounding effect of the rapid motor response present in the go trials. Finally, previous studies of inhibition in ASD used fMRI, which allows little analysis of temporal processing.
Right inferior frontal activation in adolescents with ASD

Adolescents with ASD recruited the right middle frontal gyrus in a non-homologous region (right BA 46) during the first time window of 200 to $250 \mathrm{~ms}$, unlike the adolescent controls, who first engaged the left middle frontal gyrus (BA 6). The right inferior prefrontal cortex (BA 45/46), or the ventrolateral prefrontal cortex, has consistently been shown to play an important role in inhibition [45-50]. However, children and adolescents have been found to activate the right inferior frontal gyrus less than adults [51] (Vara et al., 2013, in submission), and typical adolescents in our current study were observed to activate the right inferior cortex at sub-threshold levels. Therefore, the significant activation of this region in adolescents with ASD, while performing more poorly on task measures of inhibition (that is, the false alarm rate) than control adolescents, suggests the activation may be inefficient or ineffective in ASD due to reduced selectivity in recruitment [52,53].

\section{Reduced recruitment of non-frontal regions in adolescents with ASD}

Adolescents with ASD also showed activation in the postcentral gyrus (BA 3) at 250 to $300 \mathrm{~ms}$, which then moved 
anteriorly to the left middle frontal gyrus in BA10 from 300 to $400 \mathrm{~ms}$. Conversely, control adolescents recruited the inferior parietal lobe as well as the temporal lobe during final time window of 350 to $400 \mathrm{~ms}$. Although the recruitment of the postcentral gyrus by adolescents with ASD was parietal, the BA 3 region is associated with somatosensory-related task activity, whereas the parietal area activated by control adolescents was the inferior parietal lobe, an area commonly activated during inhibition tasks [54-57]. It is thought that parietal activity is associated with attention, or switching attentional focus within a task [58], and greater activation in this region may reflect the better task performance [57].

The poor recruitment of more widespread cortical regions, such as the parietal [31] and temporal lobes, by adolescents with ASD may explain their difficulty in executing top-down control. The poorer performance on inhibitory tasks observed in adolescents with ASD compared to controls may result from weaker behavioural regulation of a prepotent response tendency due to atypical brain activity. As the control adolescent group recruited regions outside the frontal lobes to perform the inhibition task, it is possible that adolescents with ASD were limited to frontal lobe function due to the poorer long-range connectivity reported in ASD, which in turn hinders their ability to recruit more extensive, supplementary regions $[26,52]$. The greater overall frontal activity in adolescents with ASD may be related to poor long-range connectivity and local over connectivity [59], which have been proposed to underlie deficits in at least a subgroup of individuals with ASD.

\section{BA10 activity in ASD and error monitoring}

BA 10 activity was observed between 300 to $400 \mathrm{~ms}$ in our adolescents with ASD, but activity was not seen in this region in the control adolescents. The BA 10 region has been associated with maintaining a balance between rapid responding and careful or controlled responding, during cognitive flexibility paradigms [60,61], as well as higher order mental representations of task contingencies $[62,63]$. In addition, the region within BA10 in our study corresponds to a region associated with multitasking in a meta-analysis of functional specialisation of BA 10 published by Gilbert et al. in 2006 [64]. We suggest that this area may have been recruited by our participants with ASD to process the probability of responding to the next trial, and the behavioural adjustment necessary to respond appropriately, or simply because of increased load. Adolescents with ASD had difficulty with the inhibition task, as measured by an increased false alarm rate, compared to control adolescents; it is possible that the BA 10 region was recruited to compensate for their poorer performance. Even with the activation of this compensatory region, individuals with ASD had more false alarms.
Limitations of this study include the small sample size, which limited our ability to detect between group differences beyond statistical doubt, as well as its cross sectional nature, which limits our ability to discuss developmental trajectories of the differences noted. In addition, comorbidity was not systematically assessed. This is important as inhibitory control defects are also a feature of ADHD (for example, [65,66]). Until recently, ADHD could not be considered as a comorbidity to ASD (based on DSM-IV criteria) and as such, these participants did not carry any such comorbid diagnoses. However, ADHD-like symptoms are common in this population and may complicate the picture. None of our participants were on stimulants or any other psychotropic medications, which suggests that they did not have significant ADHD.

\section{Conclusions}

Inhibition-related MEG activity in the adolescents with and without ASD demonstrated distinct spatiotemporal neural processing patterns. More extensive frontal activity was found in adolescents with ASD, which we suggest may be due to inefficient long-range connectivity, [52] as well as to short-range or local over connectivity. Our argument is supported by the poor recruitment of supplementary regions in adolescents with ASD, compared to control teens who recruited parietal and temporal areas in performance of the inhibition task. Finally, BA 10 activity was found only in adolescents with ASD, between 300 and $400 \mathrm{~ms}$, which could be interpreted as a compensatory response strategy in the ASD group [61-63] reflected in slower RTs yet higher false alarm rates. These findings highlight the atypical nature of the adolescent ASD inhibitory network, particularly in relation to the sequence of activations revealed using the temporal resolution of magnetoencephalography. We theorize that such atypicalities in inhibitory control may contribute to the social deficits of autism. Impulse control is necessary to function optimally within the framework of one's social environs and impairment in social functioning during adolescence, typically a period of tremendous development in social skills, may contribute to severe social ramifications [23]. For example, the inability to suppress socially inappropriate remarks or the failure to refrain from conversations centred on one's restricted interests, both of which are commonly observed in individuals with ASD, could lead to ostracism from peer groups. Future work needs to include large samples of longitudinal cohorts, characterized for other neurodevelopmental and neuropsychiatric comorbidities and link neurobiological findings to both associated and core symptom domains of ASD.

\section{Abbreviations}

ACC: anterior cingulate cortex; ANOVA: analysis of variance; ASD: autism spectrum disorder; BA: Brodmann area; ERB: event-related beamforming fMRI: functional Magnetic Resonance Imaging; GFP: global field power; ISI: interstimulus intervals; MEG: magnetoencephalography; IQ: intelligence 
quotient; MRI: magnetic resonance imaging; ROI: region of interest; RT: reaction time; WASI: Wechsler abbreviated scale of intelligence.

\section{Competing interests}

EA has received consultation fees from Seaside Therapeutics and Novartis, an unrestricted grant from Sanofi-Canada, and has consulted without fees to Proximagen and Neuropharm.

\section{Authors' contributions}

AV participated in study coordination, subject recruitment, data acquisition, the analyses of data and the interpretation of results and drafted the manuscript. EP assisted with interpretation of results and finalizing the manuscript. KT participated in study design, participant data acquisition, and helped with drafting of the manuscript. JV contributed to study design and interpretation of results. MT contributed to the conception and design of the study and to the interpretation of results, and drafting of the manuscript. EA participated in study design and coordination, contributed to interpretation of the results and guided the drafting of the manuscript. All authors read and approved the final manuscript.

\section{Acknowledgements}

The authors would like to thank Marc Lalancette, Hamzah Qureshi, and Travis Mills for their assistance with the analyses. The authors would also like to acknowledge Dr. Jessica Brian and the ARC team at Holland Bloorview for their efforts in characterising our sample. ASV was funded through the $U$ of T Open Fellowship Award and the work involved in this study was partially funded through the Ontario Brain Institute. Finally, the authors would like to recognize the families of the participants for their willingness to contribute a great deal of time towards research.

\section{Author details}

'Diagnostic Imaging and Neurology, The Hospital for Sick Children, University of Toronto, 555 University Avenue, M5G 1X8 Toronto, Ontario, Canada. ${ }^{2}$ Neurology, The Hospital for Sick Children, University of Toronto, 555 University Avenue, M5G 1 X8 Toronto, Ontario, Canada. ${ }^{3}$ Holland Bloorview Kids Rehabilitation Hospital, University of Toronto, 150 Kilgour road, M4G1R8 Toronto, Ontario, Canada. ${ }^{4}$ University of Toronto, 563 Spadina Crescent, Toronto M5S 2J7, Ontario, Canada. ${ }^{5}$ Paris Descartes University and UMR CNRS 3521, Paris, France. ${ }^{6}$ Bloorview Research Institute, University of Toronto, 150 Kilgour Road, Toronto, Ontario M4G 1R8, Canada.

Received: 12 June 2013 Accepted: 13 January 2014

Published: 31 January 2014

\section{References}

1. Bishop DV, Norbury CF: Executive functions in children with communication impairments, in relation to autistic symptomatology. 2: response inhibition. Autism 2005, 9:29-43.

2. Hill EL: Evaluating the theory of executive dysfunction of autism. Dev Rev 2004, 24:189-233.

3. Hill EL: Executive dysfunction in autism. Trends Cogn Sci 2004, 8:26-32.

4. American Psychiatric Association: Diagnostic and Statistical Manual of Mental Disorders (DSM-IV-TR). 4th edition. Washington, DC; 2000.

5. Aron AR: The neural basis of inhibition in cognitive control. Neuroscientist 2007, 3:214-228.

6. Miller EK, Cohen JD: An integrative theory of prefrontal cortex function. Annu Rev Neurosci 2001, 24:167-202.

7. Nyden A, Billstedt E, Hjelmquist E, Gillberg C: Neurocognitive stability in Asperger syndrome, ADHD, and reading and writing disorder: a pilot study. Dev Med Child Neurol 2001, 43:165-171.

8. Geurts HM, Verte S, Oosterlaan J, Roeyers H, Sergeant JA: How specific are executive functioning deficits in attention deficit hyperactivity disorder and autism? J Child Psychol Psych 2004, 45:836-854.

9. Luna B, Doll SK, Hegedus SJ, Minshew NJ, Sweeney J: A Maturation of executive function in autism. Biol Psychiatry 2007, 61:474-481.

10. Solomon M, Ozonoff S, Cummings N, Carter C: Cognitive control in autism spectrum disorders. Int J Dev Neurosci 2008, 26:239-247.

11. Corbett BA, Constantine $L$, Hendren R, Rocke D, Ozonoff S: Examining executive functioning in children with autism spectrum disorder, attention deficit hyperactivity disorder and typical development. Psychiat Res 2009, 166:210-222.
12. Griffith EM, Pennington BF, Wehner EA, Rogers SJ: Executive functions in young children with autism. Child Dev 1999, 70:817-832.

13. Brian JA, Tipper SP, Weaver B, Bryson SE: Inhibitory mechanisms in autism spectrum disorders: typical selective inhibition of location versus facilitated perceptual processing. J Child Psychol Psych 2003, 44:552-560.

14. Goldberg MC, Mostowsky SH, Cutting LE, Mahone EM, Astor BC, Denckla $M B$, Landa RJ: Subtle executive impairment in children with autism and children with ADHD. J Autism Dev Disord 2005, 35:279-293.

15. Lopez BR, Lincoln AJ, Ozonoff S, Lai Z: Examining the relationship between executive functions and restricted, repetitive symptoms of autistic disorder. J Autism Dev Disord 2005, 35:445-460.

16. Hill EL, Bird CM: Executive processes in Asperger syndrome: patterns of performances in a multiple case series. Neuropsychologia 2006 44:2822-2835

17. Happe F, Booth R, Charlton R, Hughes C: Executive function deficits in autism spectrum disorders and attention-deficit/hyperactivity disorder: examining profiles across domains and ages. Brain Cognition 2006, 61:25-39.

18. Kenworthy L, Yerys BE, Anthony LG, Wallace GL: Understanding executive control in autism spectrum disorders in the lab and in the real world. Neuropsychology Rev 2008, 18:320-338.

19. Ozonoff S, Strayer DL: Inhibitory function in nonretarded children with autism. J Autism Dev Disord 1997, 27:59-77.

20. Stroop JR: Studies of interference in serial verbal reactions. J Exp Psychol 1935, 18:643-662.

21. Ozonoff $\mathrm{S}$, Jensen J: Brief report: specific executive function profiles in three neurodevelopmental disorders. J Autism Dev Disord 1999, 29:171-177.

22. Tamm L, Menon V, Reiss AL: Maturation of brain function associated with response inhibition. J Am Acad Child Adolesc Psychiatry 2002, 41:1231-1238.

23. Luna B, Padmanabhan A, Hearn KO: What has fMRI told us about the development of cognitive control through adolescence? Brain Cognition 2010, 72:1-28.

24. Schmitz N, Rubia K, Daly E, Smith A, Williams S, Murphy DGM: Neural correlates of executive function in autistic spectrum disorders. Biol Psychiatry 2006, 59:7-16.

25. Kana RK, Keller TA, Minshew NJ, Just MA: Inhibitory control in high-functioning autism: decreased activation and underconnectivity in inhibition networks. Biol Psychiatry 2007, 62:198-206.

26. Just MA, Cherkassky VL, Keller TA, Kana RK, Minshew NJ: Functional and anatomical cortical underconnectivity in autism: evidence from an FMR study of an executive function task and corpus callosum morphometry. Cereb Cortex 2007, 17:951-961.

27. Thakkar KN, Polli FE, Joseph RM, Tuch DS, Hadjikhani N, Barton JJ, Manoach DS: Response monitoring, repetitive behaviour and anterior cingulate abnormalities in autism spectrum disorders (ASD). Brain 2008, 131(Pt 9):2464-2478

28. Dichter $G$, Belger $A$ : Social stimuli interfere with cognitive control in autism. Neuroimage 2007, 35:1219-1230

29. Lee PS, Yerys BE, Della-Rosa A, Foss-Feig J, Barnes KA, James JD, VanMeter J, Vaidya CJ, Gaillard WD, Kenworthy LE: Functional connectivity of the inferior frontal cortex changes with age in children with autism spectrum disorders: a fcMRl study of response inhibition. Cerebr cortex 2009, 19:1787-1794.

30. Chan AS, Han YM, Leung WW, Leung C, Wong VC, Cheung M: Abnormalities in the anterior cingulate cortex associated with attentional and inhibitory control deficits: a neurophysiological study on children with autism spectrum disorders. Res Autism Spect Dis 2011, 5:254-266.

31. Solomon M, Ozonoff SJ, Ursu S, Ravizza S, Cummings N, Ly S, Carter CS: The neural substrates of cognitive control deficits in autism spectrum disorders. Neuropsychologia 2009, 47:2515-2526.

32. Cahn BR, Polich J: Meditation states and traits: EEG, ERP, and neuroimaging studies. Psychol Bull 2006, 132:180-211.

33. Pizzagalli DA, Oakes TR, Davidson RJ: Coupling of theta activity and glucose metabolism in the human rostral anterior cingulate cortex: an EEG/PET study of normal and depressed subjects. Psychophysiology 2003, 40:939-949.

34. Hari R, Parkkonen $L$, Nangini C: The brain in time: insights from neuromagnetic recordings. Ann N Y Acad Sci 2010, 1191:89-109.

35. Vidal J, Mills T, Pang EW, Taylor MJ: Response inhibition in adults and teenagers: spatiotemporal differences in the prefrontal cortex. Brain Cognition 2012, 79:49-59. 
36. Lord C, Risi S, Lambrecht L, Cook EH, Leventhal BL, DiLavore PC, Pickles A, Rutter M: The autism diagnostic observation schedule-generic: a standard measure of social and communication deficits associated with the spectrum of autism. J Autism Dev Disord 2000, 30:205-223

37. Lord C, Rutter M, LeCouteur A: Autism diagnostic interview-revised - a revised version of a diagnostic interview for caregivers of individuals with possible pervasive developmental disorders. J Autism Dev Disord 1994, 24:659-685

38. Wechsler D: Wechsler Abbreviated Scale of Intelligence (WASI). San Antonio: Harcourt Assessment; 1999.

39. Quraan MA, Cheyne D: Reconstruction of correlated brain activity with adaptive spatial filters in MEG. Neuroimage 2010, 49:2387-2400.

40. Lalancette M, Quraan M, Cheyne D: Evaluation of multiple-sphere head models for MEG source localization. Phys Med Biol 2011, 56:5621-5635.

41. Mills T, Lalancette M, Moses SN, Taylor MJ, Quraan MA: Techniques for detection and localization of weak sources using MEG. Brain Topogr 2012, 25:248-263.

42. Quraan MA, Moses SN, Hung Y, Mills T, Taylor MJ: Detection and localization of hippocampal activity using beamformers with MEG: a detailed investigation using simulations and empirical data. Hum Brain Mapp 2011, 32:812-827.

43. Lancaster JL, Woldorff MG, Parsons LM, Liotti M, Freitas CS, Rainey L Kochunov PV, Nickerson D, Mikiten SA, Fox PT: Automated Talairach Atlas labels for functional brain mapping. Hum Brain Mapp 2000, 10:120-131.

44. Lancaster JL, Rainey LH, Summerlin JL, Freitas CS, Fox PT, Evans AC, Toga AW, Mazziotta JC: Automated labeling of the human brain: a preliminary report on the development and evaluation of a forward-transform method. Hum Brain Mapp 1997, 5:238-242.

45. Garavan H, Ross TJ, Stein EA: Right hemispheric dominance of inhibitory control: an event-related functional MRI study. Proc Natl Acad Sci U S A 1999, 96:8301-8306.

46. Kiehl KA, Liddle PF, Hopfinger JB: Error processing and the rostral anterior cingulate: an event-related fMRI study. Psychophysiology 2000, 37:216-223.

47. Konishi S, Nakajima K, Uchida I, Kameyama M, Nakahara K, Sekihara K, Miyashita Y: Transient activation of inferior prefrontal cortex during cognitive set shifting. Nature Neurosci 1998, 1:80-84.

48. Konishi S, Nakajima K, Uchida I, Kikyo H, Kameyama M, Miyashita Y: Common inhibitory mechanism in human inferior prefrontal cortex revealed by event-related functional MRI. Brain 1999, 122:981-991.

49. Liddle PF, Kiehl KA, Smith AM: Event-related fMRI study of response inhibition. Hum Brain Mapp 2001, 12:100-109.

50. Rubia K, Smith AB, Brammer MJ, Taylor E: Right inferior prefrontal cortex mediates response inhibition while mesial prefrontal cortex is responsible for error detection. Neuroimage 2003, 20:351-358.

51. Bunge SA, Dudukovic NM, Thomason ME, Vaidya CJ, Gabrieli JD: Immature frontal lobe contributions to cognitive control in children: evidence from fMRI. Neuron 2002, 33:301-311.

52. Belmonte MK, Allen G, Beckel-Mitchener A, Boulanger LM, Carper RA, Webb SJ: Autism and abnormal development of brain connectivity. J Neurosci 2004, 24:9228-9231.

53. Schulz K, Fan J, Tang C: Response inhibition in adolescents diagnosed with attention deficit hyperactivity disorder during childhood: an eventrelated fMRI Study. Am J Psychiat 2004, 9:1650-1657.

54. Rubia K, Russell T, Overmeyer S, Brammer MJ, Bullmore ET, Sharma T, Simmons A, Williams SC, Giampietro V, Andrew CM, Taylor E: Mapping motor inhibition: conjunctive brain activations across different versions of go/no-go and stop tasks. Neuroimage 2001, 13:250-261.

55. Fassbender C, Murphy K, Foxe JJ, Wylie GR, Javitt DC, Robertson IH, Garavan $\mathrm{H}$ : A topography of executive functions and their interactions revealed by functional magnetic resonance imaging. Cognitive Brain Res 2004 20:132-143.

56. Menon V, Adleman NE, White CD, Glover GH, Reiss AL: Error-related brain activation during a Go/NoGo response inhibition task. Hum Brain Mapp 2001, 12:131-143.

57. Velanova K, Wheeler ME, Luna B: Maturational changes in anterior cingulate and frontoparietal recruitment support the development of error processing and inhibitory control. Cereb cortex 2008, 18:2505-2522.

58. Corbetta M, Kincade JM, Ollinger JM, McAvoy MP, Shulman GL: Voluntary orienting is dissociated from target detection in human posterior parietal cortex. Nature Neurosci 2000, 3:292-297.
59. Courchesne E, Pierce $\mathrm{K}$ : Why the frontal cortex in autism might be talking only to itself: local over-connectivity but long-distance disconnection. Curr Opin Neurobiol 2005, 15:225-230.

60. Braver TS, Reynolds JR, Donaldson DI: Neural mechanisms of transient and sustained cognitive control during task switching. Neuron 2003, 39:713-726.

61. Brown JW, Reynolds JR, Braver TS: A computational model of fractionated conflict-control mechanisms in task-switching. Cognitive Psychol 2007, 55:37-85.

62. Badre D: Cognitive control, hierarchy, and the rostro-caudalorganization of the frontal lobes. Trends Cogn Sci 2008, 12:193-200.

63. Botvinick MM: Hierarchical models of behavior and prefrontal function. Trends Cogn Sci 2008, 12:201-208.

64. Gilbert SJ, Spengler S, Simons JS, Steele JD, Lawrie SM, Frith CD, Burgess PW: Functional specialization within the rostral prefrontal cortex (area 10): a meta analysis. J Cogn Neurosci 2006, 18:932-948.

65. Tye C, Asherson P, Ashwood KL, Azadi B, Bolton P, McLoughlin G: Attention and inhibition in children with ASD, ADHD and co-morbid ASD + ADHD: an event-related potential study. Psychol Med 2013, 15:1-16.

66. Bühler E, Bachmann C, Goyert H, Heinzel-Gutenbrunner M, Kamp-Becker I: Differential diagnosis of autism spectrum disorder and attention deficit hyperactivity disorder by means of inhibitory control and 'theory of mind'. J Autism Dev Disord 2011, 41:1718-1726.

doi:10.1186/2040-2392-5-6

Cite this article as: Vara et al:: Is inhibitory control a 'no-go' in adolescents with autism spectrum disorder? Molecular Autism 2014 5:6.

\section{Submit your next manuscript to BioMed Central and take full advantage of:}

- Convenient online submission

- Thorough peer review

- No space constraints or color figure charges

- Immediate publication on acceptance

- Inclusion in PubMed, CAS, Scopus and Google Scholar

- Research which is freely available for redistribution 